\title{
Neoplastic and Non-neoplastic Lesions of the Skull: Single-Center Experience
}

\author{
Ramazan Sarı' ${ }^{1}$, Eylem Burcu Kahraman Özlü², illhan Elmacı ${ }^{1}$ \\ ${ }^{1}$ Department of Neurosurgery, Acibadem Maslak Hospital, Istanbul, Turkey \\ ${ }^{2}$ Department of Neurosurgery, Baskent University Istanbul Hospital, Istanbul, Turkey
}

\begin{abstract}
Introduction: Tumor lesions of the skull bones are extremely rare; they are usually slow-growing but clinically aggressive. They constitute approximately $1 \%$ of all bone tumors. Although they are usually benign, malignant and metastatic lesions may also be seen. This retrospective study aimed to emphasize the importance of the histo-pathological diagnosis and surgical excision amount inplanning the treatment in advance.

Methods: In this study, the age and gender distribution of the cases, the location of the lesions, the surgical method, histopathological diagnoses and the preferred cranioplasty methods were included over 17 cases that were operated by our clinic between 2010-2020. Nine cases were male (52\%) and eight cases were female (48\%). When the diagnosis was made, the mean age was 24.3 (2 years-49 years) in the cases, while seven of the cases were children (3 girls, 4 boys). In our study, children under 17 were considered as childhood.

Results: The patients' admission symptoms were palpable pain-free mass ( $n=9)$, painful mass $(n=4)$, visual field defect and/ or vision loss related to lesion location, focal neurological deficits due to $7^{\text {th }}$ cranial nerve involvement. ( $n=1$ ), exophthalmos $(n=1)$, and one patient was an incidental diagnosis while examined for primary disease. Lesions exerted locations in the frontal bone (frontal bone and orbital roof) ( $n=9$ ), occipital bone (occipital bone and clivus) ( $n=5$ ), sphenoid bone (sphenoid wing and paracellar $(n=1)$, and lesions were multiple in two cases $(n=2)$. One patient had a diagnosis of metastatic leiomyoma before admission and one had multiple myeloma, while the other patients had no known chronic diseases.

Discussion and Conclusion: Lesions of the skull bones are rare in the clinic. Following the detailed clinical and radiological evaluation for these lesions, the total excision of the lesion to the extent possible is particularly important for the prognosis of the disease.

Keywords: Bone tumors; calvarial lesions; skull bones.
\end{abstract}

$S^{k}$ kull bones are structures that surround the brain and protect it against exogenous insults. Tumoral lesions of the skull bones are extremely rare and are approximately $1 \%$ of all bone tumors ${ }^{[1]}$. Although they are usually benign, malignant and metastatic lesions can be seen. The incidence of primary tumors metastasis to the calvarium is approximately $12 \%{ }^{[2]}$. In primary lesions, benign ones are more common than malignant ones. They are usually detected with painful or painless palpated mass or incidentally in radiological examinations $s^{[3-4]}$. The patient's age and the way of detecting the lesion are important parameters in the radiological evaluation of the lesions ${ }^{[5]}$. In most of the lesions, the diagnosis becomes clear by histopathological evaluation after radiological diagnosis, surgical removal or biopsy. The radiolog-

Correspondence (Illetişim): Ramazan Sarı, M.D. Acibadem Maslak Hastanesi, Istanbul, Turkey

Phone (Telefon): +90 5052964097 E-mail (E-posta): drramazan@windowslive.com

Submitted Date (Başvuru Tarihi): 28.07.2020 Accepted Date (Kabul Tarihi): 07.08.2020

Copyright 2020 Haydarpaşa Numune Medical Journal

OPEN ACCESS This is an open access article under the CC BY-NC license (http://creativecommons.org/licenses/by-nc/4.0/) 
ical methods used in diagnosis are direct roentgenograms, ultrasonography, computed tomography and MRI, CT-MRI angiography, their contrast studies and nuclear scintigraphic studies $^{[4]}$. In some cases, different surgical techniques and materials are used to close the defect after surgical removal. Cranioplasty to be performed in the defect area is especially important for the protection of vital neural tissue and for the protection of cranial contours for cosmetic reasons ${ }^{[6]}$. Although it is more important in the pediatric age group, closing the defect for social life or back to school provides significant benefits for both patient groups ${ }^{[7]}$. In our study, we discussed the skull bone lesions seen in the adult and pediatric patient population through histopathological diagnosis and defect repair (Figs. 1, 2).

\section{Materials and Methods}

In this study, 17 patients with calvarial lesions were treated by the same surgeon in our clinic between 2010 and 2020 . Age, gender, admission symptoms, duration of complaints, localization of lesions, cranioplasty methods in patients in need were classified through histopathological diagnoses and retrospectively.

\section{Findings}

Among the retrospectively evaluated patients, nine of the 17 cases were male (52\%) and eight were female (48\%). When the diagnosis was made, the mean age was 24.3 (2 years-49 years) in the cases, while seven of the cases were children ( 3 girls, 4 boys). In our study, children under 17 were considered as childhood. The mean age was 9.8 ( 2 years-16 years) in pediatric patients. The patients ad-

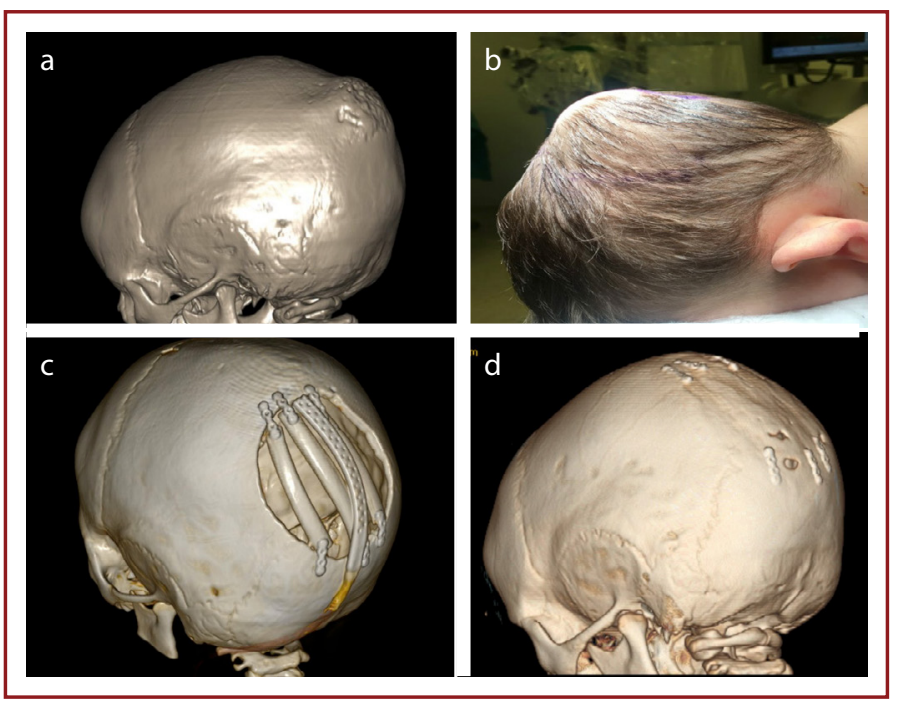

Figure 1. Intraosseous hemangioma. (a) Preop 3-D CT. (b) Perop lateral image. (c) Postop early 3-D CT. (d) Postop. 5 month control 3-D CT. mission symptomes were palpable pain-free mass $(n=9)$, painful mass $(n=4)$, visual field defect and/or vision loss related to lesion location, focal neurological deficits due to $7^{\text {th }}$ cranial nerve involvement. $(n=1)$, exophthalmus $(n=1)$, and one patient was an incidental diagnosis while examined for primary disease. Lesions exerted locations in the frontal bone (frontal bone and orbital roof) $(n=9)$, occipital bone (occipital bone and clivus) $(n=5)$, sphenoid bone (sphenoid wing and paracellar) $(n=1)$, and lesions were multiple in two cases $(n=2)$. One patient had a diagnosis of metastatic leiomyoma before admission and one had multiple myeloma, while the other patients had no known chronic diseases. Surgical excision was applied to all patients by the same surgeon. In the postoperative evaluation, 12 patients were evaluated as total and three patients were as subtotal excision, while one lesion was excised in total in two patients who had multiple lesions for diagnostic purposes. Histopathological evaluation of surgical materials revealed fibrous dysplasia $(n=2)$, intraosseous hemangioma ( $n=3$ ) (Fig. 1), ossifying fibroma $(n=2)$, osteoma $(n=3)$ aneurysmal bone cyst, $(n=1)$ langerhans cell histiocytosis (eosinophilic granuloma) $(n=2)$, epidermoid cyst $(n=1)$, intradiploic histiocytosis $(n=1)$, multiple myeloma $(n=1)$ and metastatic leiomyoma $(n=1)$ (Fig. 2).

Fibrous dysplasia cases were 1 child and 1 young adult. A 10-year-old girl patient had a complaint of a palpable progressively enlarging mass in the frontal region, which has been followed for 6 years and which was growing painless at first and then with pain for several months. The lesion
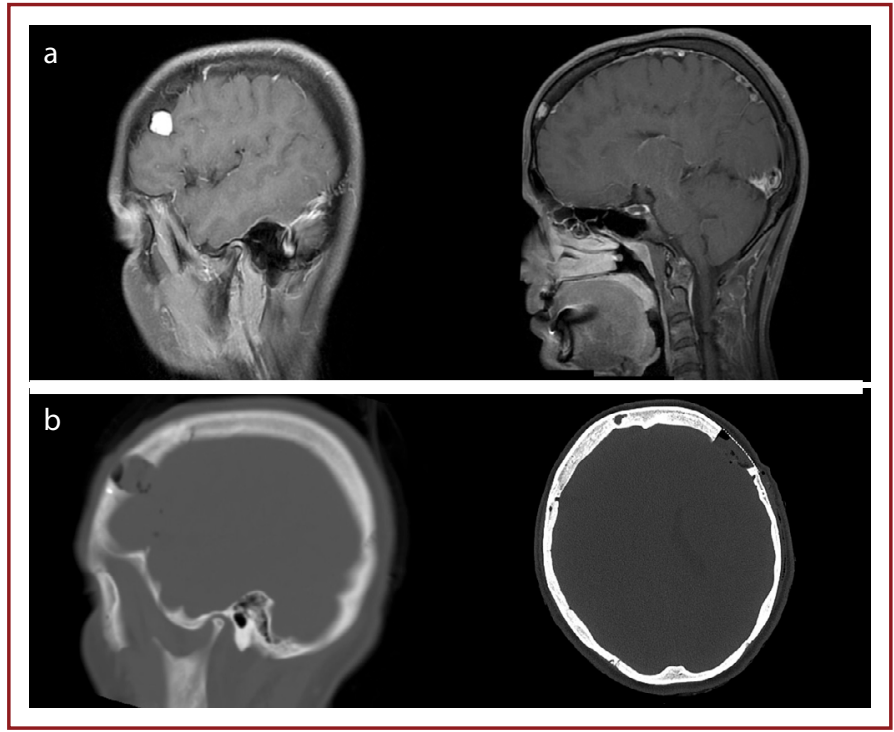

Figure 2. Leiomyoma metastasis case. (a) Preop MRI image. (b) Postop image. 
of the other case was located in the paracellular region. A young adult male patient was diagnosed as paracellar lesion two years ago after progressive vision loss in his right eye and was operated on endonasally transsphenoidal, and biopsy was performed. The patient whose biopsy result was compatible with fibrous dysplasia was followed without additional treatment. During this period, he applied to us because the patient's vision got worse. Surgical treatment was applied to both of the cases. The lesion located in the frontal bone was operated by craniectomy. Cranioplasty was applied to the craniectomy defect after total excision in the same session with acrylic graft. A case of paracellar localized fibrous dysplasia was treated with craniotomy with optic nerve decompression and subtotal mass excision. There was no increase in the deficit in the postoperative period.

Patients diagnosed with intraosseous hemangioma were two and 13 years old male and 38 years old female. A 2-year-old male patient was operated with craniotomy due to a painless swelling mass noticed by the family two months ago, and the lesion was gross totally excised. Cranioplasty was performed in the postoperative defect with a split graft taken from the $8^{\text {th }}$ rib (Fig. 1c). The systemic screenings performed by the pediatric oncology physician did not show any signs of disease, and the patient continues to follow up in the first postoperative year. In a 13-yearold male case, the operation was planned due to the mass that was noticed to grow in the left frontal region over time. The lesion was painless and hard in palpation. After microsurgical total excision, cranioplasty was applied with acrylic graft. The adult female case presented with painless swelling over the eyebrow in the frontal region, which has been growing for one year. After the total excision of the lesion with craniectomy, cranioplasty was performed with titanium mesh on the defect.

In two male adult patients with ossified fibroma, the lesions were located in the frontal region. Both patients had painless swelling for about one year. It was learned from the story that the size of the lesions increased over time. Both patients were operated by craniotomy. Cranioplasty was applied to the defect of a 41-year-old man with acrylic mesh. Since the 33-year-old male patient's lesion extending into the frontal sinus has no external tabular involvement, cranioplasty was performed with autograft and plate screw after excision of the lesion. There were three patients with osteoma, one male child, one young adult male and one female adult. A 16-year-old male patient had an exophthalmus complaint that started and increased three months ago. The orbital wall-located lesion was excised gross totally with a craniotomy. The slow progressive lesion of the 41-year-old female patient located in the occipital bone, which has been followed for about six years, was totally excised by craniotomy. Cranioplasty to the defect was performed with titanium mesh. While a 28-year-old male patient was followed up with a diagnosis of osteoma from the lesion in the same area four years ago, an operation plan was made by us due to the enlargement of the lesion. The lesion was totally excised microscopically and cranioplasty was performed to the defect with acrylic graft. A 38-year-old woman diagnosed with aneurysmal bone cyst was examined due to progressive vision loss that started two months ago, and a mass with a sphenoid wing location was detected. Due to the rapid progression of the lesion after the biopsy, it was re-operated in our clinic. Visual performance improved after the subtotal excision of the sphenoid wing mass by craniotomy.

A 5-year-old female patient with Langerhans cell histiocytosis (eosinophilic granuloma) was examined two months ago due to pain-free swelling in the left occipital region. Following the admission to our clinic, cranioplasty was performed with titanium mesh following the total excision of the lesion by microsurgery. No recurrence or residual lesions were detected at the 6th month of the patient. A 4-year-old male patient with Langerhans cell histiocytosis was diagnosed when pain and swelling were detected in the left postauricular region. The mass that destroyed the bone during follow-up with USG was operated due to increased lesion sizes. After total excision of the mass, cranioplasty was applied to the defect with titanium mesh. Systemic screening of both patients did not reveal any evidence of systemic disease.

A patient who was diagnosed with an epidermoid cyst in the right frontal bone, which was noted with painless swelling by his mother three months ago, was followed-up and enlargement of the mass and internal tabula damage was revealed in the lesion. Surgery was planned for the patient due to the progression of the lesion. Cranioplasty was applied to the microsurgical total excision site with titanium mesh. No recurrence or residual mass was observed during the first year of control of the patient. The patient, who was operated in an external center after a mass lesion located in the right frontal region detected during the examination due to headache was examined during his admission for control evaluation at the 6th postoperative month. Total excision was achieved in the second surgery of the patient for the residual lesion, who was evaluated as an eosinophilic granuloma after his first operation. Cranioplasty with metal methacrylate was applied to the craniec- 
tomy defect. In the histopathological examination of the patient, the diagnosis was confirmed as intradipleuic local histiocytosis due to the absence of Langerhans cell infiltration infiltrating compact bone and fibrous tissue, and observation of mixed infiltration of multinuclear non-Langerhans cells and rare lymphocytes. In the postoperative 5th year, the patient is followed up without complaints and recurrent lesions.

During routine controls of a patient due to multiple myeloma with spinal involvement, multiple mass lesions with calvarial localization were detected and the left frontal lesion of the patient was excised by craniectomy and cranioplasty was performed with acrylic graft. Since the pathological diagnosis was compatible with multiple myeloma, the systemic treatment continued for the primary disease.

A patient was examined for dorsalgia while she was being followed up due to uterine leiomyoma. In her examinations, a lesion with bone invasion extending to the paravertebral area at the thoracal level of 5-6 was detected and metastatic leiomyoma was detected in the biopsy. In the subsequent body scan, 4 mass lesions involving the whole tabula was observed in the calvarial bones. While following with painless lesions, the patient was interviewed at the neurooncology council, and for the purpose of diagnosis, microsurgical total excision was performed to the lesion in the left frontal location by navigation. Cranioplasty was performed on the defect with titanium mesh. Metastatic leiomyoma was detected in the histopathological evaluation of the calvarial lesion (Figs. 2 $a, b)$. Radiotherapy was applied to the lesions in the thoracic bone due to intense pain and limited mobility. Due to the progression of metastatic lesions during follow-up, the patient underwent a total abdominal hysterectomy and bilateral oophorectomy. The patient is still being followed up without any progression in the lesions in the postoperative 20 months.

\section{Discussion}

Lesions of the skull bones are rare in the clinic. Although it is thought that they constitute 0.8 to $1 \%$ of all bone tumors, some series have rates reaching $4 \%$, mostly malignant lesions ${ }^{[1]}$. These lesions are congenital, inflammatory, neoplastic lesions ${ }^{[6]}$. Calvarial lesions can be evaluated in three main groups as follows: 1) Benign lesions 2) Tumorlike lesions 3) Malignant lesions. While lesions with calvarial bone location are often recognized with painless or painful growing mass lesions, they may be presented with cranial nerve involvement findings, exophthalmos, visual field defect, headache or even changes in consciousness depending on the location of the lesion. In our cases, the symptoms of presentation were mainly painful or painless swelling. Differing from these symptoms, progressive vision loss in 1, facial paralysis in one and exophthalmos complaint in one patient were encountered. In patients with multiple myeloma and leiomyom diagnoses, the diagnosis was made during the routine controls of the primary disease.

Histopathologically, most frequently observed lesions among the calvarial lesions have been reported mainly as epidermoid/dermoid cyst and Langerhans-cell histiocytosis ${ }^{[8,9]}$. Fibrous dysplasias are benign calvarial lesions. While monocytotic and polycystic isolated forms may also be seen, they may also be encountered in association with McCune Albright syndrome. They are rare in children; the rate is approximately $2.6 \%{ }^{[4-10]}$. In our series, the rate with 1 child patient was $5.2 \%$. The patient had no additional findings indicating McCune Albright disease. Intraosseous hemangiomas are seen in all age groups. The most common lesions are observed in the frontoparietal region. In our cases, apart from one case located in the occipital region, the lesions resided in the frontal region. While they were reported to be seen most frequently in the $4^{\text {th }}$ decade, there were two children among our cases ${ }^{[4-5]}$.

Although histiocytosis X or Langerhans cell histiocytosis, formerly called an eosinophilic granuloma, is considered as a benign pathology, lesions may be presented as part of systemic disease. In the literature, cases have been reported in which this pathology shows a malignant character with rapid and progressive bone destruction. After these patients are diagnosed, surgical planning should be planned in the early period, and since there are cases with reported potential for malignant conversion, clinical monitoring should not be performed instead of surgical removal of these masses. All of our cases were evaluated for the systemic disease after the histopathological diagnosis, and no findings were observed in favor of systemic disease in any case, but patients are still being followed up ${ }^{[12]}$.

Metastatic lesions are generally observed as metastasis of malignant primary lesions. Metastases of primary lung, breast, kidney and prostate cancers are most common. Metastatic calvarial lesions have been associated with the young male age in the literature. In the study of Stark et al. ${ }^{[10]}$ evaluating 38 calvarial lesion patients, skull metastasis was detected differently in 12 patients; Wecht et al. ${ }^{[11]}$ found calvarial lesions in 42 patients over a 13 -year period ${ }^{[12]}$. While neurological deficits related 
to metastatic calvarial lesions were reported in a small number of lesions, our cases did not also have focal neurological deficits due to the lesion. One of our cases with metastatic calvarial lesions was a female patient with leiomyoma metastasis known as a primary benign disease, unlike the literature. The diagnosis of metastatic leiomyoma was confirmed after surgery applied to one of the patient-recognized calvarial lesions. It was observed that calvarial lesions remained stable after total abdominal hysterectomy and bilateral oophorectomy, which was performed, considering that it would be effective in the treatment of other systemic metastases. The patient is still being followed up stably in the $18^{\text {th }}$ postoperative month. Knowing the surgery and histopathological features of the focal calvarial lesions and learning their characteristic radiological appearance would provide the differential diagnosis of these lesions ensuring that the necessary surgical precautions will be taken beforehand ${ }^{[13]}$. When planning a surgical plan for calvarial lesions, it is important to always set the primary target as total excision. However, due to the invasion or location of the lesion into vascular structures, total excision may not be possible due to difficulties in excision. In these cases, the primary target should be determined as maximal excision of the lesion as much as possible. In our series, the total excision rate is $70.5 \%$ for 15 patients who were diagnostically excised for single lesions when the patients with multiple lesions were excluded.

In cases, it may be necessary to close the defect after excision of the lesion with various cranioplasty methods. Cranioplasty is a method used in neurosurgery practice after posttraumatic, decompressive surgery, after craniosynostosis surgeries, in patients undergoing craniectomy for tumor or other reasons. Various materials have been used over the years. While the gold standard method for cranioplasty is autologous grafts, synthetic materials are also frequently used ${ }^{[14]}$. In our patients, we used acrylic grafts in six patients and titanium mesh in six patients who needed cranioplasty after the operation, and we used costa grafts for cranioplasty in one child. Complications, such as infection due to cranioplasty, implant rejection, and implant malposition, were not observed in any of the cases. Cosmetic results, including the graft used in the facial region, were successful in patients and clinically in cranioplasties with titanium mesh.

In our clinical practice, we do not prefer autograft cranioplasty not to expose the patient to the second incision in cranioplasties performed due to tumoral lesions. In our pa- tients who need cranioplasty, we have recently performed cranioplasty with tita-nium mesh. As mentioned in the literature, we did not encounter infection complications in cases where we performed cranioplasty with titanium mesh. Titanium grafts, like acrylic grafts, are not biocompatible materials and are often preferred by us as they provide space for the growing skull in pediatric patients and a good barrier for external trauma to the parenchyma. We did not encounter any headache complaints related to titanium grafts mentioned in the literature that arise from the heat caused by the external environment ${ }^{[15,16]}$. In cases where there are no major bone defects, we think that titanium mesh and cranioplasty is a comfortable and safe option for the surgeon and the patient.

The cranioplasty with the costal graft that we applied in our 2-year-old male patient who was diagnosed with intraosseous hemangioma after the operation may be a good autograft in cases where it is not possible to split the calvarial bone. Split costa graft can be used as a good cranioplasty graft due to its low infection rate, concave shape and compatibility with the calvarium, calvarial fusion thanks to osteoblast activity and low cost ${ }^{[17]}$. When it is decided to use autografts for cranioplasty, we would like to draw attention to the importance of considering the risks of complications related to a second surgical intervention and incision. We think that with an increasing number of cases and prolonged postoperative follow-up periods, the treatment protocols of calvarial lesions can become more standardized, and multidisciplinary approaches can yield more effective clinical results in these patients.

\section{Conclusions}

Lesions of the skull bones are rare but are encountered in neurosurgical practice. Following detailed clinical and radiological evaluation for these lesions, the total excision of the lesion to the extent possible is particularly important for the prognosis of the disease. Systemic evaluation should not be ignored in cases required after histopathological diagnosis.

Ethics Committee Approval: Retrospective study.

Peer-review: Externally peer-reviewed.

Authorship Contributions: Concept: E.B.K.Ö., I.E.; Design: R.S., I.E.; Data Collection or Processing: E.B.K.Ö.; Analysis or Interpretation: R.S.; Literature Search: R.S., E.B.K.Ö.; Writing: E.B.K.Ö., R.S.

Conflict of Interest: None declared.

Financial Disclosure: The authors declared that this study received no financial support. 


\section{References}

1. Kakkar A, Nambirajan A, Suri V, Sarkar C, Kale SS, Singh M, et al. Primary Bone Tumors of the Skull: Spectrum of 125 Cases, with Review of Literature. J Neurol Surg B Skull Base 2016;77:31925. [CrossRef]

2. Arana E, Martí-Bonmatí L. CT and MR imaging of focal calvarial lesions. AJR Am J Roentgenol 1999;172:1683-8. [CrossRef]

3. Shah MV, Haines SJ. Pediatric skull, skull base, and meningeal tumors. Neurosurg Clin N Am 1992;3:893-924. [CrossRef]

4. Lloret I, Server A, Taksdal I. Calvarial lesions: a radiological approach to diagnosis. Acta Radiol 2009;50:531-42. [CrossRef]

5. Gomez CK, Schiffman SR, Bhatt AA. Radiological review of skull lesions. Insights Imaging 2018;9:857-82. [CrossRef]

6. Gibson SE, Prayson RA. Primary skull lesions in the pediatric population: a 25-year experience. Arch Pathol Lab Med 2007;131:761-6.

7. Ozgiray E, Perumal K, Cinar C, Caliskan KE, Ertan Y, Yurtseven T, et al. Management of Calvarial Tumors: A Retrospective Analysis and Literature Review. Turk Neurosurg 2016;26:690-8.

8. Cummings TJ, George TM, Fuchs HE, McLendon RE. The pathology of extracranial scalp and skull masses in young children. Clin Neuropathol 2004;23:34-43.

9. Guidetti B, Gagliardi FM. Epidermoid and dermoid cysts. Clinical evaluation and late surgical results. J Neurosurg 1977;47:12-8. [CrossRef]
10. Stark AM, Eichmann T, Mehdorn HM. Skull metastases: clinical features, differential diagnosis, and review of the literature. Surg Neurol 2003;60:219-25. [CrossRef]

11. Wecht DA, Sawaya R. Lesions of the calvaria: surgical experience with 42 patients. Ann Surg Oncol 1997;4:28-36. [CrossRef]

12. Fujimura M, Nishijima M, Umezawa K, Hayashi T, Kaimori M. A case of calvarial eosinophilic granuloma with rapid expansion and wide skull invasion: immunohistochemical analysis of $\mathrm{Ki}$ 67. J Clin Neurosci 2002;9:72-6. [CrossRef]

13. Doğan I, Kahiloğulları G, Ünlü MA. Pediatrik Hastalarda Orta Hat Yerleşimli Primer Kalvaryal Kitlelerin Cerrahi Tedavisi: Tek Merkezli Klinik Tecrübe ve Cerrahi Teknik. Van Tıp Derg 2016;23:263-8.

14. Rosinski CL, Patel S, Geever B, Chiu RG, Chaker AN, Zakrzewski J, et al. A Retrospective Comparative Analysis of Titanium Mesh and Custom Implants for Cranioplasty. Neurosurgery 2020;86:E15-E22. [CrossRef]

15. Harris DA, Fong AJ, Buchanan EP, Monson L, Khechoyan D, Lam S. History of synthetic materials in alloplastic cranioplasty. Neurosurg Focus 2014;36:E20. [CrossRef]

16. Shah AM, Jung $H$, Skirboll S. Materials used in cranioplasty: a history and analysis. Neurosurg Focus 2014;36:E19. [CrossRef]

17. Yano H, Tanaka K, Matsuo T, Tsuda M, Akita S, Hirano A. Cranioplasty with auto-purified bone flap after infection. J Craniofac Surg 2006;17:1076-9. [CrossRef] 